\title{
Synthesis of 2-aryl benzoxazoles from aldoximes
}

\begin{abstract}
Wide spectrum of biological activities of benzoxazole heterocycles aroused great interest for the development of newer methods for their synthesis. We herein report a copper catalyzed method for the synthesis of 2-aryl benzoxazoles from the reaction of aldoxime and 2-iodobromobenzene using N,N'-dimethyl ethylenediamine (DMEDA) as the ligand. The reaction proceeds with the copper-catalyzed dehydration of aldoxime leading to the nitilium ion which might be undergoing hydrolysis and subsequent $\mathrm{C}-\mathrm{O}$ bond formation in one-pot to afford 2-aryl benzoxazoles. The pure products were isolated and characterized by ${ }^{1} \mathrm{H}$ NMR, and ${ }^{13} \mathrm{C}$ NMR data.
\end{abstract}

Keywords: benzoxazoles, aldoxime, 2-iodo bromobenzene, copper catalysis, dmeda
Volume I Issue 5 - 2017

Dinesh Kumar Nayak, Niranjan Panda

Department of Chemistry, National Institute of Technology Rourkela, India

Correspondence: Niranjan Panda, Department of Chemistry, National Institute of Technology Rourkela, Odisha, India-769008, Tel +9|66 I246 2653, Email niranjanpanda@gmail.com, npanda@nitrkl.ac.in

Received: October 27, 2017 | Published: November 10, 2017

\section{Introduction}

Benzoxazoles are a class of important scaffolds and possess a wide range of applications in pharmaceutical research ${ }^{1}$ (Figure 1). Particularly, 2-substituted benzoxazole derivatives have remarkable biological potential including anticancer, antitumour and inhibitory activities. ${ }^{2-7}$ Furthermore, this motif is also abundant in several functional materials such as engineering plastics, optical brightener for textiles and metal sensors. ${ }^{8-16}$ As a result, there are increasing demands to devise versatile methods for the construction of 2-substituted benzoxazoles.

The condensation of 2-aminophenol and carboxylic acid ${ }^{17}$ or its surrogates such as aldehydes, ${ }^{18-21}$ acid chlorides, ${ }^{22-24}$ orthoesters, ${ }^{25-27}$ and $\beta$-oxodithioesters ${ }^{28}$ under various reaction conditions are the straight forward approaches to construct the benzoxazole unit. However, these methods are often associated with several limitations such as the use of highly toxic reagents, strong acids and, in some cases, harsh reaction conditions. $^{29}$ Therefore, development of suitable process for the construction of benzoxazole unit is demanding. Consequently, use of transition-metal catalyzed route offers a mild and reliable protocol to achieve the benzoxazole system with enhanced efficiency. Among the employed transition-metal catalysts, ${ }^{30-41}$ copper-catalyzed routes are considered as an ideal choice because of their commercially viability, less expensive and low cytotoxicity. ${ }^{42-50}$ Moreover, copper catalyzed benzoxazole synthesis relies on either intramolecular O-arylation of 2-halobenzanilides ${ }^{42-45}$ or direct coupling of 1,2-dihalo benzene with primary amide ${ }^{46}$ or nitrile ${ }^{47,48}$ (Figure 2). For instance, Evindar \& Batey ${ }^{42}$ reported the CuI/1,10-Phen catalyzed cyclization of orthohaloanilides to 2 -aryl benzoxazoles. Punniamurthy et al. ${ }^{43}$ used $\mathrm{CuO}-$ nanoparticle for intramolecular annulation of ortho-bromoanilide under ligand free condition to afford 2-aryl benzoxazoles. In another report, Xie et al. ${ }^{44}$ annulated the $N$-(2-iodo-/bromo-phenyl) benzamides, and even the less reactive $\mathrm{N}$-(2-chlorophenyl)benzamides, via $\mathrm{Cu}$ catalyzed intramolecular coupling to 2-aryl benzoxazoles reactions using methyl 2-methoxybenzoate as the ligand under mild reaction conditions. Similar protocol for the synthesis of benzoxazoles in aqueous medium was reported by Sanmartin et al. ${ }^{45}$ Copper-catalyzed cross-coupling of 1,2-dihaloarenes with primary amide leading to the initial formation of ortho-halo anilide and subsequent cyclization to 2-aryl benzoxazole was reported by Batey and co-workers. ${ }^{46}$ Copper-catalyzed reaction of aryl halides with nitriles leading to $\mathrm{N}$-arylamides and benzoxazoles has been developed by Xiang et al. ${ }^{47,48}$ Very recently, Dong et al. synthesized benzoxazole frameworks from the reaction of phenols and primary amines in the presence of $\mathrm{NH}_{4} \mathrm{PF}_{6}$ over copper under mild conditions using $\mathrm{O}_{2}$ as the terminal oxidant. ${ }^{49} \mathrm{CuI}$-catalyzed cyclization reactions of 2 -aminophenols with $\beta$ - diketones in the presence of Brønsted acid was also reported. ${ }^{50}$

In continuation of our earlier work on copper-catalyzed $\mathrm{N}$-aryl amide synthesis from aldoximes, ${ }^{51}$ here, we report a ligand assisted copper-catalyzed protocol for the synthesis of 2-aryl benzoxazoles from the reaction of aldoxime and 1-bromo-2-iodobenzene.

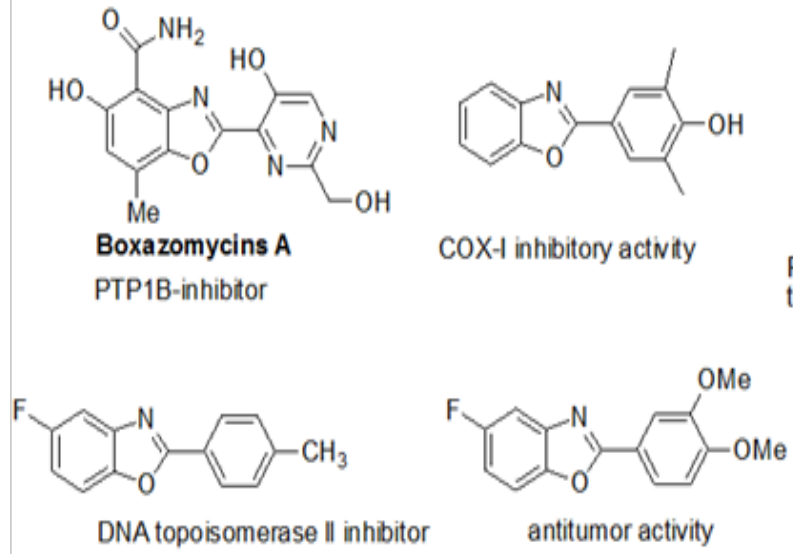

DNA topoisomerase \|l inhibitor<smiles>CCCC[C@H](CN(O)C=O)C(=O)N1CCC[C@H]1c1nc2ccccc2o1</smiles>

Peptide deformylase inhibitor of $M$. tuberculosis

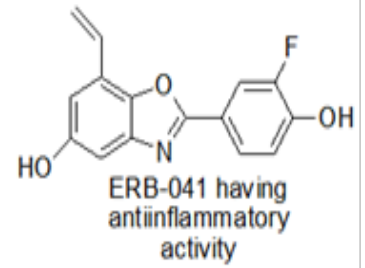

Figure I Some examples of biologically potent benzoxazoles. 


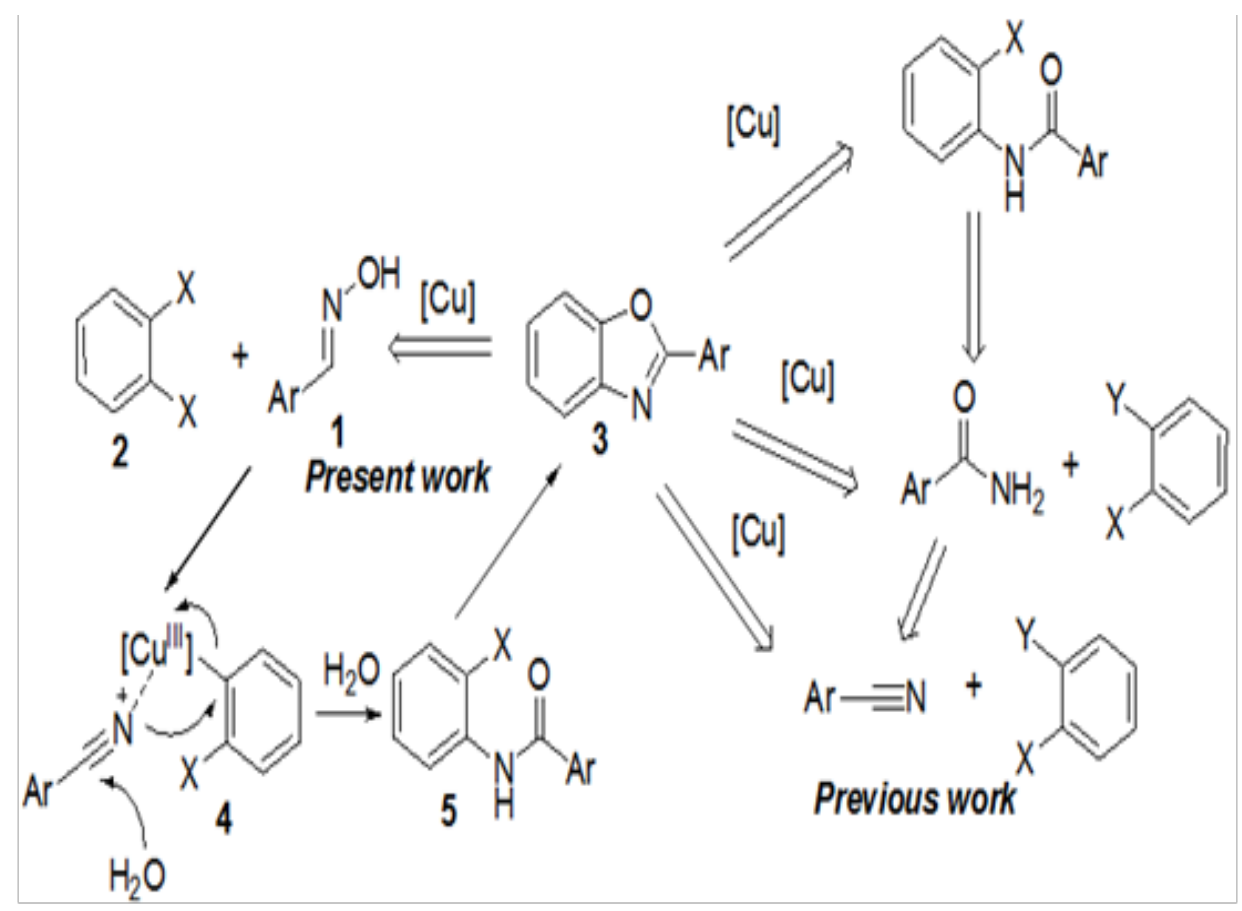

Figure 2 Copper-catalyzed 2-aryl benzoxazole synthesis.

\section{Materials and methods}

All melting points are uncorrected. All reactions were carried out in oven dried round bottom flask. Solvents and reagents were used as such without further purification. The reactions were monitored by TLC and the residue was purified by column chromatography on silica gel (Rankem, India, mess size 60-120), using an ethyl acetatepetroleum ether $\left(60-80^{\circ} \mathrm{C}\right)$ mixture as eluent. Yield of the reactions were calculated with respect to 1, 2-dihalo benzene. All NMR spectra were recorded on Bruker Avance III $\left(400 \mathrm{MHz}\right.$ for ${ }^{1} \mathrm{H}$ NMR, $100 \mathrm{MHz}$ for ${ }^{13} \mathrm{C}$ NMR) spectrometers; chemical shifts were expressed in $\delta$ units relative to TMS signal as internal reference in $\mathrm{CDCl}_{3}$ and DMSO- $\mathrm{d}_{6}$. The coupling constants ( $\mathrm{J}$ values) are expressed in Hz.

\section{General procedure for the synthesis of benzoxazole} (3a-i)

To a mixture of 1-bromo-2-iodobenzene (100mg, $0.354 \mathrm{mmol})$ and aldoxime $(1.416 \mathrm{mmol})$ in $o$-xylene $(1 \mathrm{ml}), \mathrm{K}_{2} \mathrm{CO}_{3}(1.062 \mathrm{mmol})$ and DMEDA $(0.106 \mathrm{mmol})$ was added and the mixture was heated at $140^{\circ} \mathrm{C}$ for $16 \mathrm{~h}$. After completion of the reaction, it was diluted with dichloromethane and water. The organic layer was separated and dried over anhydrous $\mathrm{Na}_{2} \mathrm{SO}_{4}$ and concentrated under reduced pressure. The crude product was purified by column chromatography using ethyl acetate and petroleum ether mixture as the eluent to get the pure product (3a-i).

\section{Results and discussion}

In our earlier report, we have described a ligand-assisted coppercatalyzed protocol for the regioselective synthesis of $\mathrm{N}$-aryl amide from the reaction of aldoxime and aryl iodide. We have proposed that in the presence of the copper catalyst, aldoxime undergo dehydration leading to intermittent nitrilium ion which subsequently passed through reductive elimination and nucleophilic attack of water or another equivalent of aldoxime to produce $\mathrm{N}$-aryl amides. Here, we speculated that, the presence of an additional halo group to the aromatic ring might induce a further reactive site for copper catalyzed $\mathrm{C}-\mathrm{O}$ bond formation to afford 2-aryl benzoxazoles (3).

At the onset, we started our investigation by the treatment of benzaldoxime 1a and 1,2-diiodobenzene (2) as the substrates to carrying out the reaction in $o$-xylene in the presence of $10 \mathrm{~mol} \%$ $\mathrm{CuSO}_{4} .5 \mathrm{H}_{2} \mathrm{O}, \mathrm{K}_{2} \mathrm{CO}_{3}$ (5 equiv), DMEDA $(30 \mathrm{~mol} \%)$ at $130^{\circ} \mathrm{C}$ for $12 \mathrm{~h}$. However, to our dismay, under this reaction condition no desired benzoxazole was produced, rather the benzaldoxime (1a) was transformed in to a mixture of benzamida and $o$-iodo benzinilide in $42 \%$ and $36 \%$ yield respectively. By changing to other metal catalyst like Cuo, copper powder, $\mathrm{CuFe}_{2} \mathrm{O}_{4}, \mathrm{CuCl}$ in both polar and non-polar solvents did not afford $\mathbf{3 a}$ rather the oxime was converted to benznitrile. However, instead of diiode benzene, when 1-bromo-2-iodobenzene (2) was treated with aldoxime in the presence of $\mathrm{CuI}(10 \mathrm{~mol} \%)$, $\mathrm{K}_{2} \mathrm{CO}_{3}$ (3 equiv), dimethyl ethylenediamine (DMEDA) (30 mol \%) in $1 \mathrm{ml}$ of o-xylene, 2-phenyl benzoxazole was produced in 52\% yield (Table 1) (Entry 1). Replacing CuI catalyst by $\mathrm{CuSO}_{4} \cdot 5 \mathrm{H}_{2} \mathrm{O}$ lesser amount (34-44\%) of 3a was produced (Entries $6 \&$ 7). Other copper catalyst including copper ferrite, copper oxide and copper chloride are less effective (Entries 5,9 \& 10). Moreover, in the absence of ligand $o$-bromo benzinilide was formed $(<15 \%)$ (Entry 2$)$. Changing the solvent to polar solvents like DMF, DMSO did not produce 3a (Entries 3 \& 4). When DMEDA was used as both ligand and base $34 \%$ of $\mathbf{3 a}$ was isolated (Entry 6). Among the tested bases (i.e. $\mathrm{K}_{2} \mathrm{CO}_{3}$, $\mathrm{KOH}, \mathrm{Cs}_{2} \mathrm{CO}_{3}$ and $\left.\mathrm{AcONa}\right) \mathrm{K}_{2} \mathrm{CO}_{3}$ (3 equiv) affords the best result. It may be noted here that lowering the reaction temperature to $100^{\circ} \mathrm{C}$ did not produce $3 \mathrm{a}$ even after a period of $36 \mathrm{~h}$.

After having established the optimum reaction conditions, we next explored the substrate scope of the CuI-catalyzed annulation reaction (Table 1). In general, different substituents such as Me, $\mathrm{OMe}, \mathrm{Cl}, \mathrm{NMe}_{2}$, on the aromatic ring of aldoxime were well tolerated to the reaction condition and provided the corresponding 2-aryl benzoxazoles in appreciable yield (Table 2). Unfortunately, electronwith drawing substituent i.e. $\mathrm{NO}_{2}$ group to the aldoxime did not produce the required benzoxazole. 
Table I Optimization of the reaction condition ${ }^{[a]}$

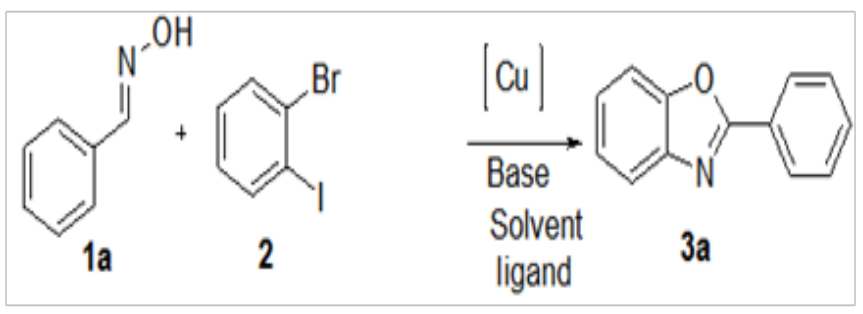

\begin{tabular}{|c|c|c|c|c|c|}
\hline Entry & Catalyst & Base & Ligand & Solvent & Yield (\%) \\
\hline I & Cul & $\mathrm{K}_{2} \mathrm{CO}_{3}$ & DMEDA & o-xylene & 52 \\
\hline 2 & Cul & $\mathrm{K}_{2} \mathrm{CO}_{3}$ & ----- & o-xylene & 0 \\
\hline 3 & Cul & $\mathrm{K}_{2} \mathrm{CO}_{3}$ & DMEDA & DMF & 0 \\
\hline 4 & Cul & $\mathrm{K}_{2} \mathrm{CO}_{3}$ & DMEDA & DMSO & 0 \\
\hline 5 & $\mathrm{CuFe}_{2} \mathrm{O}_{4}$ & $\mathrm{~K}_{2} \mathrm{CO}_{3}$ & --- & o-xylene & $<5$ \\
\hline 6 & $\mathrm{CuSO}_{4} \cdot 5 \mathrm{H}_{2} \mathrm{O}$ & DMEDA & DMEDA & o-xylene & 34 \\
\hline 7 & $\mathrm{CuSO}_{4} \cdot 5 \mathrm{H}_{2} \mathrm{O}$ & $\mathrm{K}_{2} \mathrm{CO}_{3}$ & DMEDA & o-xylene & 44 \\
\hline 8 & Cul & $\mathrm{AcONa}$ & DMEDA & DMF & 5 \\
\hline 9 & $\mathrm{CuCl}$ & $\mathrm{K}_{2} \mathrm{CO}_{3}$ & DMEDA & o-xylene & 20 \\
\hline 10 & $\mathrm{CuO}$ & $\mathrm{K}_{2} \mathrm{CO}_{3}$ & DMEDA & o-xylene & 22 \\
\hline 11 & Cul & $\mathrm{KOH}$ & I,I0-Phen & o-xylene: $\mathrm{H}_{2} \mathrm{O}(3: \mathrm{I})$ & 40 \\
\hline 12 & Cul & $\mathrm{KOH}+\mathrm{Cs}_{2} \mathrm{CO}_{3}$ (I.5 equiv each) & DMEDA & $\mathrm{H}_{2} \mathrm{O}$ (8 equiv) & 0 \\
\hline 13 & Cul & $\mathrm{Cs}_{2} \mathrm{CO}_{3}$ & I,I0-Phen & o-xylene & 47 \\
\hline
\end{tabular}

[a]Reaction condition; I-bromo-2-iodobenzene (2) ( $100 \mathrm{mg}, 0.354 \mathrm{mmol})$, benzaldoxime (Ia) (I.416 mmol), base (I.062 mmol), ligand $(0.106 \mathrm{mmol}, 30 \mathrm{~mol} \%)$, solvent $(\mathrm{Iml}), 140^{\circ} \mathrm{C}, 16 \mathrm{~h}$.

Table 2 Synthesis of 2-aryl benzoxazoles from I-bromo-2-iodobenzene [a]

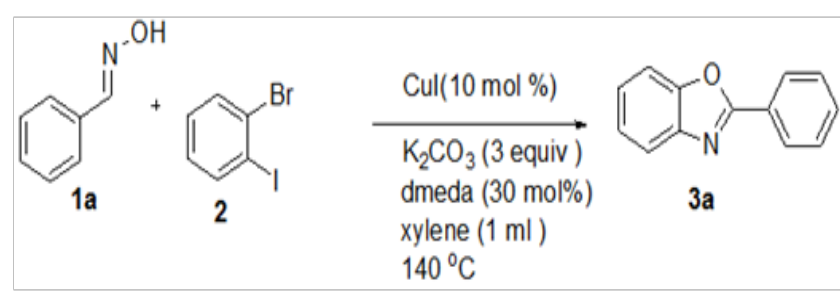

Entry


Table Continued.

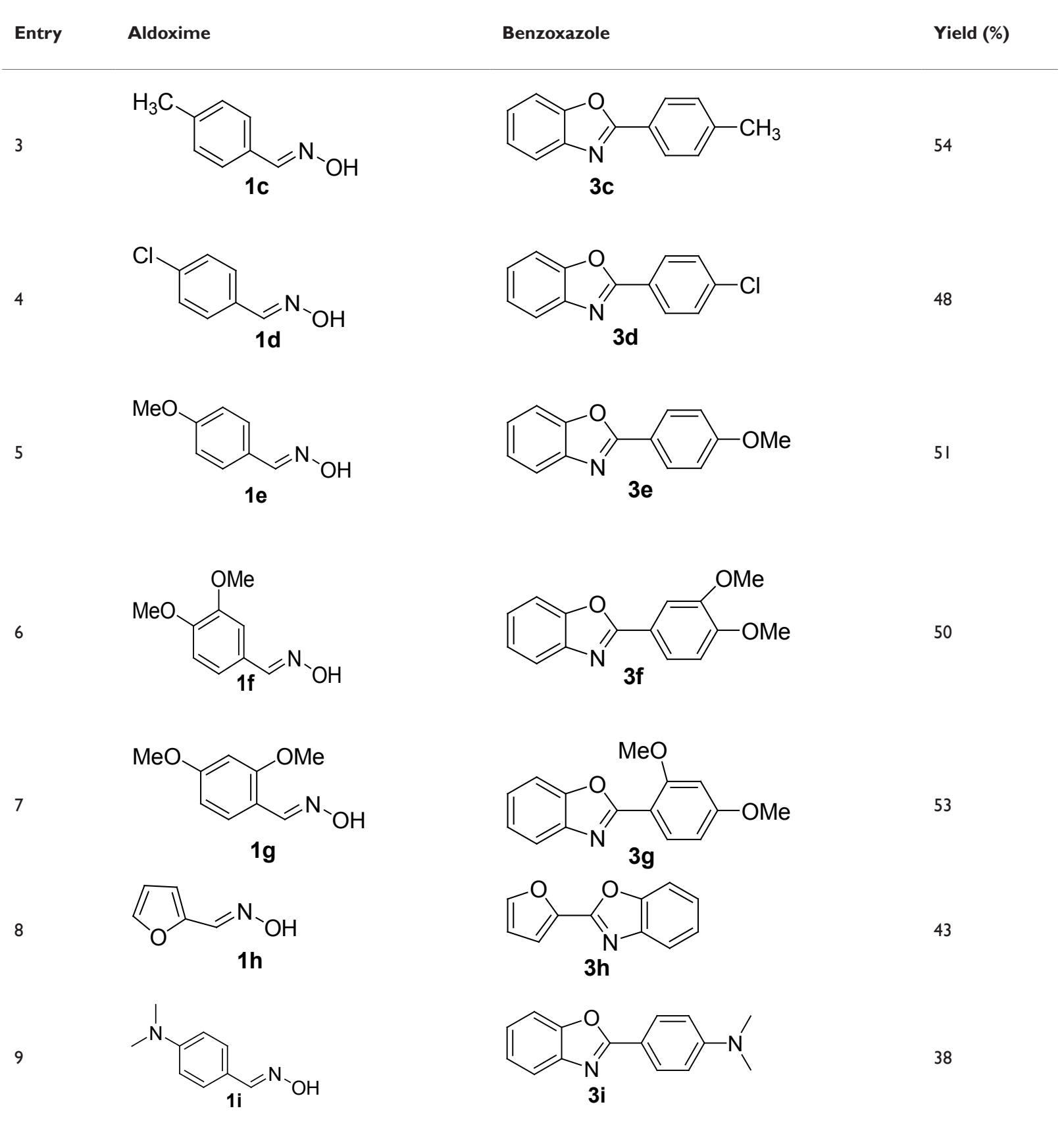

[a]Reaction condition; I-Bromo-2-iodobenzene (2) (I00mg, 0.354mmol), aldoxime (I.4I6mmol), Cul $\left(0.0354 \mathrm{mmol}^{\mathrm{a}}\right), \mathrm{K}_{2} \mathrm{CO}_{3}(\mathrm{I} .062 \mathrm{mmol})$, DMEDA (0.106mmol), o-xylene ( I ml), $140^{\circ} \mathrm{C}, 16 \mathrm{~h}$.

\section{Conclusion}

In conclusion, we have demonstrated an oxidative facial one-pot strategy for the synthesis of 2-arylbenzoxazoles in moderate yield. This reaction proceeds by using less expensive CuI-catalyst for the transformation of aryl aldoxime to 2-aryl benzoxazoles in the presence of 1-bromo-2-iodobenzene.

\section{Summary of spectroscopic data}

i. 2-phenyl benzo[d] oxazole $^{52}$ (3a): Yellowish crystalline solid; m. p. $100-102^{\circ} \mathrm{C} .{ }^{1} \mathrm{H}$ NMR $\left(400 \mathrm{MHz}, \mathrm{CDCl}_{3}\right) \delta 8.32-8.25$ (m,
2H), 7.83 - $7.77(\mathrm{~m}, 1 \mathrm{H}), 7.64$ - $7.58(\mathrm{~m}, 1 \mathrm{H}), 7.58$ - $7.51(\mathrm{~m}, 3 \mathrm{H})$, $7.38\left(\mathrm{dd}, 2 \mathrm{H}, \mathrm{J}_{1}=6 \mathrm{~Hz}, \mathrm{~J}_{2}=3.2 \mathrm{~Hz}\right) ;{ }^{13} \mathrm{C}$ NMR $\left(100 \mathrm{MHz}, \mathrm{CDCl}_{3}\right)$ 8163.0, 150.7, 142.1, 131.5, 128.9, 127.6, 125.1, 124.5, 120.0, 110.6 .

ii. 2-(2-chlorophenyl) benzo [d] $\operatorname{oxazole}^{53}$ (3b): White crystalline solid; m. p. $63-65^{\circ} \mathrm{C} .{ }^{1} \mathrm{H}$ NMR $\left(400 \mathrm{MHz}, \mathrm{CDCl}_{3}\right) \delta 8.16(\mathrm{dd}, 1 \mathrm{H}$, $\left.\mathrm{J}_{1}=7.2 \mathrm{~Hz}, \mathrm{~J}_{2}=1.8 \mathrm{~Hz}\right), 7.91-7.84(\mathrm{~m}, 1 \mathrm{H}), 7.67-7.55(\mathrm{~m}, 2 \mathrm{H}), 7.51-$ $7.36(\mathrm{~m}, 4 \mathrm{H}) ;{ }^{13} \mathrm{C} \mathrm{NMR}\left(100 \mathrm{MHz}, \mathrm{CDCl}_{3}\right) \delta 160.9,150.5,141.6$, $133.4,131.9,131.8,131.4,126.9,125.6,124.6,120.5,110.7$.

iii. 2-(p-tolyl) benzo [d] $\operatorname{oxazole}^{53}(3 \mathrm{c})$ : Yellowish white crystalline 
solid; m. p. 88-90 ${ }^{\circ} \mathrm{C} .{ }^{1} \mathrm{H}$ NMR (400MHz, $\left.\mathrm{CDCl}_{3}\right): \delta 8.16(\mathrm{~d}, 2 \mathrm{H}$, $\mathrm{J}=3.48 \mathrm{~Hz}), 7.81-7.75(\mathrm{~m}, 1 \mathrm{H}), 7.61-7.53(\mathrm{~m}, 1 \mathrm{H}), 7.40-7.30(\mathrm{~m}$, $4 \mathrm{H}), 2.44$ (s, $3 \mathrm{H}):{ }^{13} \mathrm{C}$ NMR $\left(100 \mathrm{MHz}, \mathrm{CDCl}_{3}\right): \delta 163.3,150.6$, $142.1,142.0,129.6,127.5,124.8,124.5,124.3,119.8,110.5$, 21.6.

iv. 2-(4-chlorophenyl) benzo [d] $\operatorname{oxazole}^{52}$ (3d): White crystalline solid; m. p. $144-145^{\circ} \mathrm{C} .{ }^{1} \mathrm{H}$ NMR $\left(400 \mathrm{MHz}, \mathrm{CDCl}_{3}\right): \delta 8.21$ (d, $2 \mathrm{H}, \mathrm{J}=8.4 \mathrm{~Hz}), 7.82-7.76(\mathrm{~m}, 1 \mathrm{H}), 7.63-7.57(\mathrm{~m}, 1 \mathrm{H}), 7.52(\mathrm{~d}$, $2 \mathrm{H}, \mathrm{J}=8.8 \mathrm{~Hz}), 7.42-7.26(\mathrm{~m}, 2 \mathrm{H}):{ }^{13} \mathrm{C} \mathrm{NMR}\left(100 \mathrm{MHz}, \mathrm{CDCl}_{3}\right)$ : $\delta 162.0,150.7,141.9,137.7,129.3,128.8,125.6,125.3,124.7$, 120.0, 110.6.

v. 2-(4-methoxyphenyl) benzo [d] $\operatorname{oxazole}^{52}$ (3e): White crystalline solid; m. p. $126-128^{\circ} \mathrm{C} .{ }^{1} \mathrm{H}$ NMR $\left(400 \mathrm{MHz}, \mathrm{CDCl}_{3}\right): \delta$ $8.21(\mathrm{~d}, 2 \mathrm{H}, \mathrm{J}=9.2 \mathrm{~Hz}), 7.79-7.72(\mathrm{~m}, 1 \mathrm{H}), 7.60-7.53(\mathrm{~m}, 1 \mathrm{H})$, 7.39-7.29 (m, 2H), 7.04 (d, 2H, J=8.8Hz), $3.90(\mathrm{~s}, 3 \mathrm{H}):{ }^{13} \mathrm{C}$ NMR $\left(100 \mathrm{MHz}, \mathrm{CDCl}_{3}\right): \delta 163.1,162.3,160.6,142.2,129.3,124.6$, 124.4, 119.6, 119.6, 114.3, 110.3, 55.4.

vi. 2-(3,4-dimethoxyphenyl) benzo[d] $\operatorname{oxazole}^{54}(3 \mathrm{f})$ : White crystalline solid; m. p. $109-110^{\circ} \mathrm{C} .{ }^{1} \mathrm{H}$ NMR $\left(400 \mathrm{MHz}, \mathrm{CDCl}_{3}\right) \delta 7.88$ $\left(\mathrm{dd}, 1 \mathrm{H}, \mathrm{J}_{1}=8.4 \mathrm{~Hz}, \mathrm{~J}_{2}=\mathrm{Hz}\right), 7.80-7.53(\mathrm{~m}, 2 \mathrm{H}), 7.61-7.55(\mathrm{~m}, 1 \mathrm{H})$, 7.38-7.32 (m, 2H), $7.01(\mathrm{~d}, 1 \mathrm{H}, \mathrm{J}=8.4 \mathrm{~Hz}), 4.04(\mathrm{~s}, 3 \mathrm{H}), 3.99(\mathrm{~s}$, $3 \mathrm{H}) ;{ }^{13} \mathrm{C} \mathrm{NMR}\left(100 \mathrm{MHz}, \mathrm{CDCl}_{3}\right) \delta 163.1,151.9,150.7,149.2$, $142.2,124.7,124.5,121.2,119.6,111.0,110.4,110.0,56.1,56.0$.

vii. 2-(2,4-dimethoxyphenyl) benzo[d] oxazole $^{55}(3 \mathrm{~g})$ : Yellowish white crystalline solid; m. p. $58-60^{\circ} \mathrm{C} .{ }^{1} \mathrm{H}$ NMR $(400 \mathrm{MHz}$, $\left.\mathrm{CDCl}_{3}\right) \delta 8.11(\mathrm{~d}, 1 \mathrm{H}, \mathrm{J}=8.4 \mathrm{~Hz}), 7.84-7.75(\mathrm{~m}, 1 \mathrm{H}), 7.59-7.52(\mathrm{~m}$, $1 \mathrm{H}), 7.36-7.26(\mathrm{~m}, 2 \mathrm{H}), 6.66-6.58(\mathrm{~m}, 2 \mathrm{H}), 4.01(\mathrm{~s}, 3 \mathrm{H}), 3.88(\mathrm{~s}$, $3 \mathrm{H}) ;{ }^{13} \mathrm{C}$ NMR $\left(100 \mathrm{MHz}, \mathrm{CDCl}_{3}\right) \delta 163.5,161.6,159.9,150.0$, $142.2,132.4,124.4,124.1,119.8,110.2,109.0,105.2,99.1,56.1$, 55.6 .

viii. 2-(furan-2-yl) benzo[d] oxazole $^{52}(3 \mathrm{~h})$ : Yellowish white crystalline solid; m. p. 107-108 ${ }^{\circ} \mathrm{C} .{ }^{1} \mathrm{H}$ NMR $\left(400 \mathrm{MHz}, \mathrm{CDCl}_{3}\right)$ : 87.81- $7.74(\mathrm{~m}, 1 \mathrm{H}), 7.69(\mathrm{~d}, 1 \mathrm{H}, \mathrm{J}=8 \mathrm{~Hz}), 7.61-7.55(\mathrm{~m}, 1 \mathrm{H})$, 7.41- $7.34(\mathrm{~m}, 2 \mathrm{H}), 7.32-7.26(\mathrm{~m}, 1 \mathrm{H}), 6.67-6.61(\mathrm{~m}, 1 \mathrm{H}) ;{ }^{13} \mathrm{C}$ NMR $\left(100 \mathrm{MHz}, \mathrm{CDCl}_{3}\right): \delta 155.4,150.1,145.7,142.6,141.6$, 125.3, 124.8, 120.1, 114.2, 112.2, 110.6.

ix. 4-(benzo[d]oxazol-2-yl)-N,N-dimethylaniline ${ }^{52} \quad(3 \mathrm{i})$ : White crystalline solid; m. p. $185-187^{\circ} \mathrm{C} .{ }^{1} \mathrm{H}$ NMR $\left(400 \mathrm{MHz}, \mathrm{CDCl}_{3}\right)$ : $\delta 8.13\left(\mathrm{dd}, 2 \mathrm{H}, \mathrm{J}_{1}=7.2 \mathrm{~Hz}, \mathrm{~J}_{2}=2 \mathrm{~Hz}\right), 7.72\left(\mathrm{dd}, 1 \mathrm{H}, \mathrm{J}_{1}=6.8 \mathrm{~Hz}\right.$, $\left.\mathrm{J}_{2}=1.6 \mathrm{~Hz}\right), 7.57-7.51(\mathrm{~m}, 1 \mathrm{H}), 7.36-7.24(\mathrm{~m}, 2 \mathrm{H}), 6.81-6.74(\mathrm{~m}$, $2 \mathrm{H}), 3.07(\mathrm{~s}, 6 \mathrm{H}) ;{ }^{13} \mathrm{C}$ NMR $\left(100 \mathrm{MHz}, \mathrm{CDCl}_{3}\right): 164.2,152.3$, $150.5,142.5,129.0,124.1,123.9,119.0,114.1,111.5,110.1$, 40.1 .

\section{Acknowledgements}

Authors are thankful to SERB, DST, Govt. of India (Grant No. SB/ S1/OC-21/2014), DST) for financial support.

\section{Conflict of interest}

The author declares no conflict of interest.

\section{References}

1. Demmer CS, Bunch L. Benzoxazoles and oxazolopyridines in medicinal chemistry studies. Eur J Med Chem. 2015;97:778-785.
2. Dunwell DW, Evans D, Hicks TA. Synthesis and antiinflammatory activity of some 2-heteroaryl-.alpha.-methyl-5-benzoxazoleacetic acids. J Med Chem. 1975;18(11):1158-1159.

3. Oksuzoglu E, Tekiner-Gulbas B, Alper S, et al. Some benzoxazoles and benzimidazoles as DNA topoisomerase I and II inhibitors. J Enzyme Inhib Med Chem. 2008;23(1):37-42.

4. Oksuzoglu E, Temiz-Arpaci O, Tekiner-Gulbas B, et al. A study on the genotoxic activities of some new benzoxazoles. Med Chem Res. 2007;16(1):1-14.

5. Potashman MH, Bready J, Coxon A, et al. Design, synthesis, and evaluation of orally active benzimidazoles and benzoxazoles as vascular endothelial growth factor-2 receptor tyrosine kinase inhibitors. $J$ Med Chem. 2007;50(18):4351-4373.

6. Huang ST, Hsei IJ, Chen C. Synthesis and anticancer evaluation of bis(benzimidazoles), bis(benzoxazoles), and benzothiazoles. Bioorg Med Chem. 2006;14(7):6106-6119.

7. Kumar D, Jacob MR, Reynolds MB, et al. Synthesis and evaluation of anticancer benzoxazoles and benzimidazoles related to UK-1. Bioorg Med Chem. 2002;10(12):3997-4004.

8. Yang CC, Tian Y, Chen CY, et al. A novel benzoxazole-containing poly(N-isopropylacrylamide) copolymer as a multifunctional sensing material. Macromol Rapid Commun. 2007;28(7):894-899.

9. Ogura T, Yamaguchi K, Shibasaki Y, et al. Photosensitive Poly(Benzoxazole) Based on Poly(O-Hydroxy Amide), Dissolution Inhibitor, Thermo Acid Generator, and Photo Acid Generator. Polym J. 2007;39(3):245251.

10. Ogoshi T, Miyake J, Chujo Y. Multiresponsive Photopatterning OrganicInorganic Polymer Hybrids Using a Caged Photoluminescence Compound. Macromolecules. 2005;38(10):4425-4431.

11. Park S, Kim S, Seo J, et al. Strongly Fluorescent and Thermally Stable Functional Polybenzoxazole Film: Excited-State Intramolecular Proton Transfer and Chemically Amplified Photopatterning. Macromolecules. 2005;38(11):4557-4559.

12. Leaver IH, Milligan B. Fluorescent whitening agents - a survey (197482). Dyes Pigm. 1984;5(2):109-144.

13. Majumdar KC, Ghosh T, Rao DSS, et al. Unsymmetrical tetracatenar liquid crystals containing 2-phenylbenzoxazole:Synthesis and characterization. Liq Cryst. 2013;40(3):305-313.

14. Chen $\mathrm{P}, \mathrm{Xu} \mathrm{Y}, \mathrm{Du} \mathrm{W}$, et al. New mesogenic compounds containing a terminal-substituted benzoxazole unit. Mol Crys Liq Cryst. 2014;592(1):44-62.

15. Shavaleev NM, Scopelliti R, Gumy F, et al. Benzothiazole- and benzoxazole-substituted pyridine-2-carboxylates as efficient sensitizers of europium luminescence. Inorg Chem. 2009;48(13):6178-6191.

16. Oliveira E, Genovese D, Juris R, et al. Bioinspired systems for metal-ion sensing: new emissive peptide probes based on benzo[d]oxazole derivatives and their gold and silica nanoparticles. Inorg Chem. 2011;50(18):8834-8849.

17. Ying-Hung So, Heeschen JP. Mechanism of Polyphosphoric Acid and Phosphorus Pentoxide-Methanesulfonic Acid as Synthetic Reagents for Benzoxazole Formation. J Org Chem. 1997;62(11):3552-3561.

18. Kawashita Y, Nakamichi N, Kawabata H, et al. Direct and Practical Synthesis of 2-Arylbenzoxazoles Promoted by Activated Carbon. Org Lett. 2003;5(20):3713-3715.

19. Chang J, Zhao K, Pan S. Synthesis of 2-arylbenzoxazoles via DDQ promoted oxidative cyclization of phenolic Schiff bases-a solution-phase strategy for library synthesis. Tetrahedron Lett. 2002;43(6):951-954. 
20. Rajender SV, Dilip K. Manganese triacetate oxidation of phenolic Schiff bases: synthesis of 2-arylbenzoxazoles. J Heterocycl Chem. 1998;35(6):1539-1540.

21. Padalkar VS, Gupta VD, Phatangare KR, et al. Indion 190 resin, efficient, environmentally friendly, and reusable catalyst for synthesis of benzimidazoles, benzoxazoles, and benzothiazoles. Green Chem Lett Rev. 2012;5(2):139-145.

22. Goldstein SW, Dambek PJ. A facile synthesis of methyl 2-substituted-4 benzoxazolecarboxylates. J Heterocyclic Chem. 1990;27(2):335-336.

23. Shoar RH, Heidary M, Farzaneh M, et al. Synthesis of benzoxazoles catalyzed by MCM-41, a green and reusable catalyst. Synth Commun. 2009;39(10):1742-1751.

24. Nadaf RN, Siddiqui SA, Daniel T, et al. Room temperature ionic liquid promoted regioselective synthesis of 2-aryl benzimidazoles, benzoxazoles and benzthiazoles under ambient conditions. J Mol Catal A Chem. 2004;214(1):155-160

25. Jekins GL, Knevel AM, Davis C. Notes. New synthesis of the benzothiazole and benzoxazole rings. J Org Chem. 1961;26(1):274-274.

26. Kunz KR, Taylor EW, Hutton HM, et al. A simple and convenient synthesis of 5-substituted benzoxazoles. Org Prep Proc Int. 1990;22(5):613618 .

27. Bastug G, Eviolitte C, Marko IE. Functionalized orthoesters as powerfu building blocks for the efficient preparation of heteroaromatic bicycles. Org Lett. 2012;14(13):3502-3505.

28. Srivastava A, Shukla G, Singh MS. p-Toluenesulfonic acid-catalyzed metal-free formal [4 + 1] heteroannulation via N-H/O-H/S-H functionalization: One-pot access to $2-$ aryl/hetaryl/alkyl benzazole derivatives. Tetrahedron. 2017;73(7):879-887.

29. Peng J, Zong C, Ye M, et al. Direct transition-metal-free intramolecular $\mathrm{C}-\mathrm{O}$ bond formation: synthesis of benzoxazole derivatives. Org Biomol Chem. 2011;9(4):1225-1230.

30. Ramachandran R, Prakash G, Selvamuruga S, et al. Efficient and versatile catalysis of N-alkylation of heterocyclic amines with alcohols and one-pot synthesis of 2-aryl substituted benzazoles with newly designed ruthenium (II) complexes of PNS thiosemicarbazones. Dalton Trans. 2014;43(21):7889-7902.

31. Shibahara F, Yamaguchi E, Murai T. Direct multiple C-H bond arylation reaction of heteroarenes catalyzed by cationic palladium complex bearing 1,10-phenanthroline. Chem Commun (Camb). 2014;46(14):24712473.

32. Boissarie PJ, Hamilton ZE, Lang S, et al. A Powerful Palladium-Catalyzed Multicomponent Process for the Preparation of Oxazolines and Benzoxazoles. Org Lett. 2011;13(23):6256-6259.

33. Neumann KT, Lindhardt AT, Andersen BB, et al. Access to 2-(Het) aryl and 2-Styryl Benzoxazoles via Palladium-Catalyzed Aminocarbonylation of Aryl and Vinyl Bromides. Org Lett. 2015;17(9):2094-2097.

34. Yoo JW, Yuan H, Miyamura H, et al. Facile Preparation of 2-Substituted Benzoxazoles and Benzothiazoles via Aerobic Oxidation of Phenolic and Thiophenolic Imines Catalyzed by Polymer-Incarcerated Platinum Nanoclusters. Adv Synth Catal. 2011;353(17):3085-3089.

35. Saha P, Ali MA, Ghosh P, et al. Cobalt-catalyzed intramolecular C-N and $\mathrm{C}-\mathrm{O}$ cross-coupling reactions:synthesis of benzimidazoles and benzoxazoles. Org Biomol Chem. 2010;8(24):5692-5699.

36. Guo T. Palladium/copper-catalyzed cross-coupling reactions for the synthesis of 4-heteroaryl quinolinone. Tetrahedron Letters. 2016;57(51):5837-5840.
37. Kuroda K, Tsuyumine S, Kodama T. Direct Synthesis of a PDE4 Inhibitor by Using $\mathrm{Pd}-\mathrm{Cu}-\mathrm{Catalyzed} \mathrm{C}-\mathrm{H} / \mathrm{C}-\mathrm{Br}$ Coupling of Benzoxazole with a Heteroaryl Bromide. Org Process Res Develop. 2016;20(6):10531058.

38. Lin WH, Wu WC, Selvaraju M, et al. One-pot synthesis of benzazoles and quinazolinones via iron pentacarbonyl mediated carbonylation of aryl iodides under microwave irradiation. Org Chem Front. 2017;4(3):392-397.

39. Wu M, Hu X, Liu J, et al. Iron-catalyzed 2-arylbenzoxazole formation from o-nitrophenols and benzylic alcohols. Org Lett. 2012;14(11):27222725 .

40. Bonnamour J, Bolm C. Iron-catalyzed intramolecular o-arylation:synthesis of 2-aryl benzoxazoles. Org Lett. 2008;10(13):2665-2667.

41. Tang L, Guo X, Yang Y, et al. Gold nanoparticles supported on titanium dioxide:an efficient catalyst for highly selective synthesis of benzoxazoles and benzimidazoles. Chem Commun (Camb). 2014;50(46):61456148 .

42. Evindar G, Batey RA. Parallel Synthesis of a Library of Benzoxazoles and Benzothiazoles Using Ligand-Accelerated Copper-Catalyzed Cyclizations of ortho-Halobenzanilides. J Org Chem. 2006;71(5):18021808.

43. Saha P, Ramana T, Purkait N, et al. Ligand-Free Copper-Catalyzed Synthesis of Substituted Benzimidazoles, 2-Aminobenzimidazoles, 2-Aminobenzothiazoles, and Benzoxazoles. J Org Chem. 2009;74(22):87198725 .

44. Wu F, Zhang J, Wei Q, et al. Copper-catalyzed intramolecular O-arylation:a simple and efficient method for benzoxazole synthesis. Org Biomol Chem. 2014;12(47):9696-9701.

45. Barbero N, Carril M, Sanmartin R, et al. Copper-catalyzed intramolecular O-arylation of aryl chlorides and bromides:a straightforward approach to benzo[d]oxazoles in water. Tetrahedron. 2007;63(42):1042510432

46. Viirre RD, Evindar G, Batey RA. Copper-Catalyzed Domino Annulation Approaches to the Synthesis of Benzoxazoles under Microwave-Accelerated and Conventional Thermal Conditions. J Org Chem. 2008;73(9):3452-3459.

47. Xiang SK, Zhang DX, Hu H, et al. An improved protocol for synthesis of $\mathrm{N}$-arylamides and benzoxazoles by the copper-catalyzed reaction of aryl halides with nitriles. Tetrahedron. 2013;69(47):10022-10029.

48. Xiang SK, Zhang DX, Hu H, et al. Synthesis of N-Arylamides by Copper-Catalyzed Amination of Aryl Halides with Nitriles. Adv Synth Catal. 2013;355(8):1495-1499.

49. Liu L, Qian LW, Wu S, et al. Selective Aerobic C-H Amination of Phenols with Primary Amines over Copper toward Benzoxazoles. Org Lett. 2017;19(11):2849-2852.

50. Mayo MS, Yu X, Zhou X, et al. Synthesis of Benzoxazoles from 2-Aminophenols and $\beta$-Diketones Using a Combined Catalyst of Bronsted Acid and Copper Iodide. J Org Chem. 2014;79(13):6310-6314.

51. Panda N, Mothkuri R, Nayak DK. Copper-Catalyzed Regioselective Synthesis of N-Aryl Amides from Aldoximes and Aryl Halides. Euro J Org Chem. 2014;2014(8):1602-1605

52. Gao F, Kimb BS, Walsh PJ. Room-temperature palladium-catalyzed direct 2-arylation of benzoxazoles with aryl and heteroaryl bromides. Chem Commun (Camb). 2014;50(73):10661-10664.

53. Maleki B, Baghayeri M, Vahdat SM, et al. Ag@ $\mathrm{TiO}$, nanocomposite; synthesis, characterization and its application as a novel and recyclable catalyst for the one-pot synthesis of benzoxazole derivatives in aqueous media. RSC Adv . 2015:5(58):46545-46551. 
54. Ferguson DM, Rudolph SR, Kalyani D. Palladium-Catalyzed Intra- and Intermolecular C-H Arylation Using Mesylates:Synthetic Scope and Mechanistic Studies. ACS Catal. 2014;4(7):2395-2401.
55. Alagille D, Baldwin RM, Tamagnan GD. One-step synthesis of 2-arylbenzothiazole (BTA) and -benzoxazole precursors for in vivo imaging of $\beta$-amyloid plaques. Tetrahedron Lett. 2005;46(8):1349-1351. 